\title{
Educación Superior Inclusiva y Autonomía Pedagógica: Análisis en Dos Contextos Iberoamericanos
}

\section{Inclusive Higher Education and Pedagogical Autonomy: Analysis of Two Ibero-American Contexts}

\author{
Marta Medina-García ${ }^{1}$, Lina Higueras-Rodríguez ${ }^{2}, \mathrm{M}^{\mathrm{a}}$ del Mar García-Vita ${ }^{2, *}$ \\ ${ }^{1}$ Universidad de Jaén, España \\ ${ }^{2}$ Universidad de Almería, España
}

\section{DESCRIPTORES:}

Autonomía pedagógica

Educación inclusiva

Responsabilidad social universitaria

Calidad educativa

Educación superior

\section{RESUMEN}

Conseguir que las instituciones educativas respondan a las demandas sociales y los imperativos legales como seña de calidad y excelencia es un reto presente en la actualidad. Este trabajo tiene como objetivo conocer la relación entre autonomía pedagógica e inclusión en el ámbito de la educación superior universitaria sobre dos realidades iberoamericanas: la española y la colombiana, con el propósito de asentar una base teórica en relación con la temática de estudio. Para ello se utilizó como método la revisión teórico-reflexiva a partir de una búsqueda exhaustiva en la base de datos Web of Science. Los hallazgos señalan cómo la autonomía pedagógica actúa como elemento promotor de la inclusión como una cuestión de calidad educativa y responsabilidad social, a pesar de que existe una desconexión entre la normativa y su implementación práctica, poniéndose de manifiesto la necesidad de una formación del profesorado y el establecimiento de un diseño universal en pro de la educación superior inclusiva como parte del ejercicio de su autonomía pedagógica institucional. Las implicaciones de este trabajo para las instituciones de educación superior iberoamericanas conllevan cambios tanto en la gestión como en las políticas educativas.

\section{KEYWORDS:}

Pedagogical autonomy

Educational inclusion

University social

responsibility

Educational quality

Higher education

\section{ABSTRACT:}

Getting educational Ensuring that educational institutions respond to social demands and legal requirements as a sign of quality and excellence is a current challenge. This work aims to understand the relationship between teaching autonomy and inclusion in the field of higher education in two Latin American realities: the Spanish and Colombian, in order to establish a theoretical basis in relation to the subject of study. To this end, the theoretical-reflexive review was used as a method based on an exhaustive search in the Web of Science database. The findings point out how pedagogical autonomy acts as a promoter of inclusion as a question of educational quality and social responsibility, despite the fact that there is a disconnect between the regulations and their practical implementation, highlighting the need for teacher training and the establishment of a universal design for Inclusive Higher Education as part of the exercise of institutional pedagogical autonomy. The implications of this work for Ibero-American higher education institutions involve changes in both management and educational policy.

CÓMO CITAR:

Medina-García, M., Higueras-Rodríguez, L. y García-Vita, M. M. (2021). Educación superior inclusiva y autonomía pedagógica: Análisis en dos contextos iberoamericanos. REICE. Revista Iberoamericana sobre Calidad, Eficacia y Cambio en Educación, 19(2), 55-72. https://doi.org/10.15366/reice2021.19.2.004

*Contacto: margvita@ual.es

ISSN: 1696-4713

www.rinace.net/reice

revistas.uam.es/reice
Recibido:

19 de septiembre 2020

$1^{\text {a }}$ Evaluación: 22 de diciembre 2020

2a Evaluación: 7 de febrero 2021

Aceptado: 22 de febrero 2021 


\section{Introducción}

La sociedad actual está caracterizada por la diversidad de opciones personales, sociales y culturales. Desde la Declaración Universal de los Derechos Humanos (Naciones Unidas, 1948) se especifica que la educación es un derecho que responde en igualdad de condiciones con los demás derechos. Es ahí donde se hace imprescindible la actuación de diferentes agentes, entre ellos los educativos, con el fin de garantizar la igualdad de oportunidades, equidad e inclusión. Para que se lleve a cabo una educación inclusiva, se necesita de profesionales comprometidos con la realidad educativa, y de centros que dispongan de autonomía pedagógica que diseñe planes y programas que den respuesta a situaciones reales y que garanticen la inclusión de todo el alumnado (Casanova, 2020). Lo que pretende la educación inclusiva es no dejar a nadie fuera del sistema pues cada persona pertenece y tiene responsabilidades dentro de un grupo (Ainscow, 2001; Ainscow y West, 2008; Stainback y Stainback, 1999). Por tanto, lo que se propone desde la educación inclusiva es la transformación de las aulas y las instituciones educativas con el fin de garantizar una inclusión real y mejorar la calidad de los procesos educativos.

Sobre estas premisas la autonomía se debe perfilar como un indicador esencial de la calidad educativa o lo que es lo mismo, garante del modelo de inclusión, en detrimento de los imperantes en la actualidad como el capacitismo, que domina los criterios de evaluación y calidad de los sistemas educativos tal y como recoge el Observatorio Estatal de la Discapacidad (2019).

La autonomía pedagógica se concreta a nivel de las enseñanzas medias a través del proyecto educativo y en el ámbito de la educación superior en los estatutos universitarios y demás acciones universitarias en las que se fijan los valores, los objetivos y las prioridades educativas. De la misma manera se aborda como una cuestión interesante dentro del campo de la formación del profesorado para promover la mejora de su desarrollo profesional docente (Medina-García, 2018; Olivencia y Hernández, 2012; Pérez Gómez, 1998). Por tanto, no hay duda de la coherencia, viabilidad e idoneidad de que esta cuestión se aborde desde la educación superior universitaria como marco de la implementación de la inclusión educativa (Almenta y Muñoz, 2007; Casanova, 2020).

A pesar de que desde este trabajo se pone en valor la relación o exigencia de la autonomía pedagógica para garantizar la educación inclusiva universitaria, son escasos los trabajos e investigaciones que relacionan estos elementos, de ahí la novedad y aportación de este trabajo, el cual tiene como objetivo principal conocer la relación entre autonomía pedagógica e inclusión en el ámbito de la educación superior universitaria sobre dos realidades iberoamericanas: la española y la colombiana, con el propósito de asentar una base teórica en relación con la temática de estudio. Para ello, se centró en el análisis del contexto universitario español y colombiano en materia de inclusión y autonomía pedagógica, a través del estudio de lo recogido en trabajos teóricos, normativa y legislación, exposición de experiencias institucionales y trabajos de investigación previos.

Por todo ello, se hace imprescindible analizar la autonomía pedagógica y sus implicaciones para el desarrollo de la educación inclusiva. Como punto de partida, se plantean las siguientes cuestiones: ¿los centros disponen de esa autonomía para abarcar el tema de la inclusión? ¿Qué se propone desde el ámbito universitario? La educación inclusiva es el punto de partida para que alumnado, familia y toda la comunidad educativa pueda conseguir niveles de logro, iniciando procesos de cambio e innovación desde la diversificación curricular, y consiguiendo una autonomía pedagógica eficaz y necesaria para dar respuesta educativa desde una perspectiva inclusiva.

El presente trabajo se estructura en torno a un método de revisión bibliográfica exploratoria y descriptiva de la literatura permitiendo la obtención de un panorama general sobre el conocimiento existente en la cuestión a tratar. Por un lado, se obtuvo la evolución temporal de la autonomía pedagógica y su incidencia en la educación inclusiva; y, por otro lado, las posibles temáticas emergentes en relación al ámbito universitario.

Si se hace un análisis conceptual, la sistematización de investigaciones y experiencias educativas es una parte imprescindible de la fundamentación teórica, y ésta a su vez, se sustenta desde el paradigma cualitativo. Todo ello, ayuda a explorar diferentes contextos, intervenciones y sujetos para lograr distintas descripciones y explicaciones de la realidad subjetiva de la acción (Mesa et al., 2000; Strauss y Corbin, 1998). 
Para comenzar, se inició una búsqueda de documentos donde se tuvieron en cuenta una serie de descriptores, extraídos del tesauro de ERIC, que describiese a la perfección la intención de búsqueda y el objeto de estudio.

Los descriptores fueron: "Autonomía educativa", "Educación inclusiva”, "Universidad". Tras la sección de éstos, se procedió a la búsqueda de documentos en la base de datos Web of sciences (WOS) a través de la formulación de una educación de búsqueda para que ésta fuera más eficaz y tuviera una mayor rigurosidad. La fórmula fue: TS = (Educational autonomy AND Inclusive education AND university). Tras su aplicación, se tuvo en cuenta una serie de criterios de inclusión, entre los que destacan: a) Estudios correspondientes al área de investigación "Education educational research" y "Special education"; b) Que fueran estudios realizados en los últimos 10 años (2010-2020). Tras los filtros de búsqueda, se obtuvieron 7 resultados que ayudaron a conocer el estado de la cuestión de forma general y para poder abarcar la investigación conociendo como punto de partida lo que se había trabajado anteriormente, haciendo hincapié en temas que no se han ido abarcando con una mayor rigurosidad previamente.

Con esto, se pretende dar a conocer el procedimiento llevado a cabo para la elaboración del trabajo teórico-reflexivo que se presenta. No se trata de una investigación que plasma meramente los datos de una revisión sistemática que explora el tema propuesto, la autonomía pedagógica y a la educación inclusiva dentro del ámbito universitario, sino que desemboca en valoraciones e interpretaciones sobre el estado de la cuestión a nivel de la práctica, de la teoría, de la investigación y de la normativa y política.

\section{Conceptualizando la autonomía pedagógica en el marco de la educación general}

Las continuas reformas educativas a las que está sometido el sistema educativo español, han ido promoviendo innovaciones relacionadas a modelos de descentralización y autonomía, ya que un mayor nivel de autonomía contribuye a la mejora y éxito escolar (Ávila et al., 2019; López et al., 2016; Torres, 2018).

Algunas de las características principales para el logro de la calidad y mejora de los centros educativos son la autonomía pedagógica, organizativa y de gestión.

El eje central de este trabajo se centró en la autonomía pedagógica. Esta se define en la Ley Orgánica 8/2013, de 9 de diciembre, para la mejora de la calidad educativa (en adelante, LOMCE) como la capacidad que tienen los centros educativos para adoptar acuerdos, "planes de trabajo, tipos de organización, normas de convivencia y ampliación del calendario escolar o del horario lectivo de distintas áreas o materias, en los términos que establezcan las Administraciones educativas y dentro de las posibilidades que permita la normativa aplicable, incluida la laboral, sin que, en ningún caso, se impongan aportaciones a las familias ni exigencias para las Administraciones educativas" (LOMCE, 2013, p.71). Es la Ley Orgánica 2/2006, de 3 de mayo, de Educación (en adelante, LOE) quien consolida el principio de autonomía de los centros educativos en sus tres vertientes, organizativa, gestión y pedagógica. "Los centros docentes dispondrán de autonomía para elaborar, aprobar y ejecutar un proyecto educativo y un proyecto de gestión, así como las normas de organización y funcionamiento del centro” (LOE, 2006, p.71). Además, se especifican los principios relacionados con la educación inclusiva. Se destaca "la equidad, que garantice la igualdad de oportunidades, la inclusión educativa y la no discriminación y actúe como elemento compensador de las desigualdades personales, culturales, económicas y sociales, con especial atención a las que deriven de discapacidad" (LOE, 2006, p.14) y "la autonomía para establecer y adecuar las actuaciones organizativas y curriculares en el marco de las competencias y responsabilidades que corresponden al Estado, a las Comunidades Autónomas, a las corporaciones locales y a los centros educativos" (LOE, 2006, p. 15).

Es por ello que la autonomía, de manera global, y la pedagógica en particular, es una vía para que se desarrolle y garantice la inclusión y coordinen actuaciones hacia la educación inclusiva, equitativa y de calidad. Con el fin de garantizar dichas actuaciones, se necesita de profesionales comprometidos que lideren los centros educativos.

Para que exista un verdadero aprendizaje del alumnado y la mejora del funcionamiento del centro, se debe tener en cuenta el desarrollo de una autonomía pedagógica y el modelo de los directivos escolares. 
Esto quiere decir que lo que se pretende es fomentar un liderazgo pedagógico o centrado en el aprendizaje (learning-centred leadership). Lo que propone Bolívar (2010a; 2010b; 2019) en sus investigaciones es que, a través de este tipo de liderazgo, el foco de atención se haga en el currículum y en la pedagogía, dejando a un lado la gestión y administración. Es decir, lo que se propone es que en los centros educativos se promueva el desarrollo profesional del docente y una mejora en el aprendizaje (Casanova, 2012; Desimone, 2007; Robinson et al., 2009). En investigaciones como las de Martínez-Valdivia y otros (2018) y Moral y otros (2020) se habla de cómo diferentes centros educativos disponen de ese tipo de liderazgo y cómo se desarrolla una mejora escolar en términos de inclusión con la creación de proyectos educativos inclusivos.

Desde la perspectiva de la autonomía de gestión y administración, distintos estudios nacionales e internacionales delimitan el trabajo de la dirección escolar como imprescindible y reconocido en la mejora de los centros en particular, y en la mejora del sistema y calidad en el proceso educativo (Fullan, 2011; Leithwood et al., 2006; Louis y Wahlstrom, 2011; Ng, 2011; Rodríguez et al., 2013; Sarasúa, 2013; Segura et al., 2020; Silva et al., 2018).

La autonomía profesional llega a ser un concepto difícil de definir, pero fácil de distinguir cuáles son los rasgos significativos del profesional de la enseñanza que realiza su trabajo autónomo y los factores que facilitan o inhiben esa autonomía (Ávalos, 1994).

Desde una perspectiva comunitaria, la autonomía pedagógica a través de un liderazgo educativo debe situar el aprendizaje en el centro de la vida escolar (Day et al., 2016; Niemi et al., 2018), realizando planteamientos participativos (Diamond y Spillane, 2016; Martínez-Valdivia et al., 2018).

La LOMCE menciona que desde la OCDE recomiendan el aumento de la autonomía de los centros para la mejora de los resultados de éstos. Aunque se le dé un valor muy importante a la autonomía, los datos internacionales siguen mostrando que existe un déficit en el sistema educativo español. Se hace necesario que cada centro pueda identificar sus fortalezas y debilidades, con el fin de adaptar su proceso de enseñanza al contexto que le rodea. Se debe tener en cuenta las necesidades que presente el alumnado y todo el contexto del centro educativo. Tal como se menciona en la LOMCE,

la autonomía de los centros es una puerta abierta a la atención a la diversidad de los alumnos y alumnas, que mantiene la cohesión y unidad del sistema y abre nuevas posibilidades de cooperación entre los centros y de creación de redes de apoyo y aprendizaje compartido. (p. 7)

Se considera que, los centros educativos, desde el marco de su autonomía pedagógica y organizativa, pueden establecer actuaciones de intervención educativa inclusiva como garantía de equidad e inclusión de todo el alumnado a través de la disposición de recursos, colaboración, trabajo en equipo, participación activa, etc. Para ello pueden llevar a cabo un proyecto educativo inclusivo que favorezca la diversidad (Bolívar, 2018; Casanova, 2016; Parcerisa, 2016).

\section{Aproximación a la autonomía pedagógica en el ámbito universitario}

La Comisión Europea y un número significativo de gobiernos europeos han reconocido la necesidad de la autonomía universitaria. En su Comunicación "Cumplir con la agenda de modernización de las Universidades: educación, investigación e innovación” (mayo de 2006), la Comisión Europea marca como prioridad la creación de nuevos marcos para las Universidades, caracterizados por una mayor autonomía y responsabilidad. El Real Decreto 1393/2007, de 29 de octubre, por el que se establece la ordenación de las enseñanzas universitarias oficiales, confirma este enfoque y establece un vínculo explícito entre la autonomía y la capacidad de las Universidades para responder a las expectativas de la sociedad. La relevancia de la autonomía para las Universidades se pone de manifiesto en el Informe de Tendencias 2010 de la Asociación Europea de Universidades, donde casi la mitad de los encuestados (43\%) valoran los aspectos propios de la autonomía, como uno de los eventos más importantes de la institución universitaria de los últimos años (Sursock y Smidt, 2010). Este hallazgo coincide con investigaciones previas (Aghion et al., 2008; Estermann y Bennetot, 2011; Reichert y Tauch, 2005). 
A pesar de esta apuesta clara y unánime, la autonomía universitaria se entiende de manera muy diferente en cada contexto, estando estrechamente relacionada con las tendencias legislativas, de ordenación territorial y realidades socioculturales de cada país. Por eso, podemos hablar de diversidad de modelos de autonomía universitaria incluso dentro de Europa. La tradición universitaria en cada país hace que las percepciones y la terminología asociadas a la autonomía universitaria varíen significativamente (Estermann et al., 2011). Por esos cambios constantes cuesta generar un marco de referencia para su medición objetiva.

En un intento de permitir un análisis comparativo entre países en un contexto europeo, la Declaración de Lisboa del año 2010 de la Asociación Europea de Universidades (2010) (EUA según sus siglas en inglés) establece cuatro dimensiones básicas de autonomía en el ámbito universitario. Se complementa dicha clasificación con la descripción y análisis realizado por el estudio de Estermann y otros (2011) de estos ámbitos:

Autonomía académica o pedagógica: implica decidir sobre el proceso de enseñanza, la oferta y estructura de títulos, planes de estudio, currículo y metodología; así como el número y selección de estudiantes. Además, en cuanto a la investigación y generación de conocimiento, significa tener capacidad de decidir sobre áreas prioritarias, alcance, objetivos y métodos de investigación. De manera más concreta, los indicadores para valorar la autonomía pedagógica a nivel de cada universidad propuestos por Estermann y otros (2011) son: capacidad para decidir sobre el número total de estudiantes, capacidad para seleccionar estudiantes, capacidad para introducir y terminar programas de grado, capacidad para elegir el idioma de instrucción, capacidad para seleccionar mecanismos de aseguramiento de la calidad, capacidad para seleccionar proveedores de garantía de calidad y capacidad para diseñar el contenido de las carreras.

En la mayoría de los países europeos, las Universidades son esencialmente libres para desarrollar su perfil académico y curricular, aunque la introducción de nuevos programas generalmente requiere alguna forma de aprobación por parte del ministerio competente o de otra autoridad pública y, a menudo, está vinculada a negociaciones presupuestarias. El acceso de estudiantes normalmente está regulado a nivel nacional en la mayoría de los países europeos (tanto los requisitos como el número de estudiantes) dejando poca autonomía en ese sentido a las instituciones:

Autonomía financiera: implica todo lo concerniente en materia presupuestaria, patrimonial o gestión financiera. Algunas de las manifestaciones de esta autonomía son el poder adquirir y asignar fondos, decidir sobre las tasas de matrícula y tener la capacidad de acumular excedentes.

Autonomía organizativa: algunos elementos de dicha autonomía son el establecimiento de estructuras universitarias, la elaboración de estatutos, la celebración de contratos o la elección de órganos y personas decisorias. En general, las Universidades tienen más autonomía en cuanto a las estructuras administrativas que la académica.

Autonomía de la dotación de personal: lo relativo a la responsabilidad de contratación de personal, los salarios y ascensos. Este ámbito está íntimamente relacionado con la autonomía financiera y con la organizacional.

Aunque el objeto de este trabajo es centrarse en la autonomía pedagógica no se puede ignorar el resto de las dimensiones ya que, como se puede apreciar, existen fuertes interrelaciones entre las diferentes áreas de autonomía. Si las Universidades se ven limitadas en su faceta financiera, otras dimensiones de la autonomía como la autonomía organizativa o la académica pueden verse severamente afectadas; por lo cual legislar o considerarlas aisladamente puede carecer de sentido (Estermann, 2015; Estermann et al., 2011; Vilaverde, 2020). Es por ello que se han planteado estudios empíricos que han demostrado que la capacidad de una Universidad para generar ingresos adicionales se relaciona con el grado de autonomía institucional en todas sus dimensiones otorgado por el marco regulatorio en el que opera (Estermann y Bennetot, 2011; Garces y Letelier, 2016).

Además de esa fuerte interrelación, se debe hablar de otros trabajos que han apostado por poner de manifiesto que la autonomía universitaria ayuda a mejorar los estándares de calidad, entendiendo la formulación de políticas que reforman la autonomía como un motor importante de la modernización universitaria (Estermann, 2015). Se dice que la autonomía pedagógica, en términos generales, se percibe como un elemento clave para la promoción y renovación de las Universidades europeas, así como para garantizar 
la calidad de las mismas (Estermann et al., 2011; González y De León, 2011). Otros, han sido más concretos y manifiestan un vínculo entre el desempeño universitario y la autonomía institucional (De Boer et al. 2010; Jongbloed et al., 2010). Un par de años después de la Declaración de Lisboa, la EUA en la Declaración de Praga (2009) presentaron 10 factores de éxito para las Universidades europeas entre los que incluyeron la autonomía, además, vinculándolo con el deber ser de las instituciones universitarias de servir mejor a la sociedad y contar con líderes con autonomía universitaria para diseñar estructuras internas eficientes, dar forma a programas académicos y utilizar recursos financieros.

Sin embargo, no se debe entender el ejercicio de la autonomía universitaria como algo que entre en confrontación con el derecho a la libertad de cátedra de los docentes, ya que "los derechos de libertad de cátedra y autonomía universitaria no son incompatibles ni se autoexcluyen, sino que se complementan" (Fernández Vivas, 2017, p. 79). De hecho, en el artículo 2.3 de la Ley Orgánica 6/2001, de 21 de diciembre, de Universidades (en adelante, LOU) se define la autonomía universitaria como aquella que tiene sus cimientos en "el principio de libertad académica, que se manifiesta en las libertades de cátedra, de investigación y de estudio" (p.14).

Cuando se habla de autonomía universitaria no se refiere a cambiar el poder de manos de un estado o ente gubernamental a manos de cargos o líderes propios de la institución universitaria, tampoco necesariamente a convertir las Universidades en empresas autónomas regidas por reglas del mercado, sino que hay considerar que la naturaleza de la autonomía está estrechamente relacionada con el papel que desempeñan las Universidades en la sociedad, sin que repercuta en unas áreas de conocimiento en detrimento de otras (Fumasoli et al., 2014; Jiménez Delgado et al., 2016; Kerr, 2001; Kezar y Eckel, 2004, Olsen y Maassen, 2007). Un modelo de dirección institucional exige normas y valores sociales, según los cuales, las Universidades son autónomas según su propio papel e historia, por los que la educación superior y la investigación son entes independientes que permiten que funcionen de manera independiente de un Estado (Fumasoli et al., 2014). La autonomía además permite que las instituciones sean dinámicas y puedan competir a escala internacional, promoviendo el desarrollo de un liderazgo de tipo emprendedor (Kerr, 1993), sin que dicha autonomía se convierta en un elemento pasivo, sino que debe asumirse de manera activa (Carrillo y Flores, 2013; Kerr, 2001).

Por otro lado, si se hace referencia a la formación del profesorado y la autonomía, esta se hace patente en la reflexión, el pensamiento y la toma de decisiones en torno al desarrollo de la actividad docente (Olivencia y Hernández, 2012). Para Salinas (1994) y Stenhouse (1998), esto se resume en el proceso de planificación, en el cual el docente analiza y se para a pensar cómo atender a sus estudiantes. Una cuestión que no es ajena al ámbito universitario (Nielsen, 2010; Ocampo, 2013).

\subsection{Autonomía pedagógica: un recurso al servicio de la responsabilidad social univer- sitaria y la inclusión}

La relevancia de la Universidad en la sociedad ha aumentado, a medida que la educación superior masiva se ha convertido en una realidad y el papel de la Universidad en el estímulo de la innovación y el crecimiento económico se ha destacado cada vez más en las políticas nacionales (Fumasoli et al., 2014). Las Universidades son cada vez más amplias y socialmente inclusivas, mostrando un cambio en la noción del conocimiento hacia la práctica y lo profesional, y dando muestra de una mayor flexibilidad organizativa y mayor cercanía con la sociedad, diversificando sus redes y actividades con los actores públicos y privados (Christensen, 2011; Maasen, 2008; Paradeise et al., 2009a, 2009b).

La institución universitaria debe tener muy presente su compromiso y los planteamientos de responsabilidad social universitaria que suponen una reformulación de la Universidad conforme a valores, retos, formas de organización y de gestión hacia iniciativas que supongan más responsabilidad con la sociedad y su desarrollo más sostenible y equitativo, siguiendo los objetivos de transferencia del conocimiento conforme a la ética y el compromiso social (González et al., 2015).

La United Nations Educational, Scientific and Cultural Organization (Unesco en adelante) en la Declaración Mundial sobre la Educación Superior para el siglo XXI de 1998 (Unesco, 1998), trata sobre la responsabilidad social universitaria entendiéndose como aquella responsabilidad de las Universidades para atender las necesidades y demandas sociales promoviendo esa responsabilidad en los estudiantes. Y este 
aspecto se reafirma con la instauración del Espacio Europeo de Educación Superior que viene a reforzar la misión de la Universidad socialmente responsable (Vega, 2009).

Asimismo, la responsabilidad social de la Universidad supone un compromiso con aspectos como la inclusión, los cuales se traducen en la práctica en indicadores como los siguientes que señala Vega (2009), instalaciones y servicios para estudiantes con discapacidad; más formación en derechos sociales; facilidad de acceso a materiales y recursos docentes para todos los estudiantes; estudios e investigación acerca de la inclusión social a nivel local y comunitario; fortalecimiento de capacidades y competencias básicas de los sectores en situaciones especiales.

En definitiva, supone dotarla de las herramientas necesarias para lograr una Universidad acorde a las necesidades y exigencias del siglo XXI (Martínez, 2017), una gestión y un liderazgo eficaz y eficiente, y una experiencia técnica y especializada renovada en una variedad de áreas según las demandas que se plantean. Entre estas demandas incipientes, qué duda cabe que se encuentra ofrecer una respuesta adecuada a la diversidad presente en el aula y eliminar los obstáculos que impidan lograr este hecho, lo que supone una transformación de la cultura, las políticas y las prácticas de las instituciones educativas para garantizar el derecho a la educación inclusiva (García, 2020). Esta cuestión debe ser tratada de forma conjunta, tanto por las Universidades como por las autoridades públicas pertinentes (Estermann, 2015). Y por supuesto, se debe tener presente que las características que adopte la responsabilidad social universitaria, como parte de la filosofía y cultura de gestión y gobierno de la institución universitaria, va a depender fundamentalmente del ejercicio que hagan las mismas de su autonomía (Núñez y Alonso, 2009).

El compromiso de las instituciones educativas y concretamente universitarias, con el desarrollo social en el marco de la inclusión educativa, se pone en entredicho con los resultados de investigaciones como la de Fedulova y otros (2019) que indican déficits relevantes en la formación y las prácticas inclusivas lo que supone dificultades para lograr el desarrollo sostenible de la sociedad.

De la misma forma, este deber u obligación social de las Universidades con la inclusión se vincula necesariamente con el legal, pues debe garantizar la presencia, participación y progreso de sus estudiantes, ofreciendo un sistema educativo de calidad e inclusivo actuando así conforme a lo previsto en las normas en materia de educación y discapacidad que rigen los sistemas educativos, así como a los preceptos que rigen el propio proceso de inclusión (Medina-García, 2017).

Cuando se habla de educación inclusiva el objetivo que se pretende es el de fomentar la cohesión social y la equidad y lograr su implementación en todas las etapas educativas incluida la universitaria (Llorent et al., 2020). Pero lo cierto es que para garantizar la inclusión educativa en el ámbito universitario es fundamental la coordinación de los recursos humanos que la componen (Ainscow y Miles, 2008). De forma específica el profesorado universitario es esencial en este proceso debido a la autonomía de las Universidades en la gestión, docencia e investigación, de ahí que su compromiso y formación con la educación inclusiva sea vital para el logro e implementación de esta cuestión (Moriña et al., 2014). No obstante, lo cierto es que los docentes disfrutan de poca autonomía (Olivencia y Hernández, 2012). De manera que formación y autonomía se convierten en elementos entroncados (Almenta y Muñoz, 2007) claves para la inclusión, pero actualmente actúan como barrera para su implementación.

Como señala Casanova (2020) dependiendo del ejercicio de la autonomía que hagan las instituciones educativas superiores se ofrecerá o no una respuesta adecuada a los estudiantes, en cuyo caso no se puede obviar que la autonomía pedagógica supone adecuar el currículum a las necesidades del contexto y la población. De manera que la autonomía se convierte en un factor de calidad indiscutible que va de la mano de la inclusión.

No obstante, a pesar del interés por garantizar la educación inclusiva las investigaciones al respecto siguen siendo escasas e insuficientes (Llorent et al., 2020) al igual que ocurre con la multitud de iniciativas y acciones propuestas, aún es un objetivo pendiente de culminación en el sistema educativo en general, y, de forma particular, en la educación superior universitaria. Por ello es necesario que la Universidad sea consciente de su compromiso pedagógico real con la universalización del derecho a la educación, así como con la calidad de la misma, lo que supone entre otras cuestiones, generar acciones concretas ante la diversidad para dar una respuesta adecuada a sus necesidades, garantizando así la accesibilidad y la adquisición de conocimientos y competencias mediante el uso de estrategias metodológicas innovadoras (Jaimes et al., 2009). 


\section{Educación superior inclusiva y autonomía pedagógica: dos realidades diferentes en el contexto iberoamericano}

La educación como proceso eminentemente socializador posibilita la interacción entre sujetos que presentan diferencias propias del ser humano y que no pueden obviar los sistemas educativos (Gómez y García-Vita, 2017). Sobre esta premisa se basa el derecho a la educación inclusiva el cual debe implementarse en todas las etapas del sistema educativo, incluida la educación superior (Jaimes et al., 2009), tal y como contempla la normativa internacional como la Convención Internacional de los Derechos de las personas con discapacidad del 13 de diciembre de 2006 (Naciones Unidas, 2016) (en adelante Convención). Sin embargo, a pesar de lo objetivo de la situación planteada, la realidad del sistema educativo dista mucho de garantizar este derecho (Alcaín y Medina-García, 2017; Jaimes et al., 2009).

A continuación, se centró el análisis en el contexto universitario español y colombiano en materia de inclusión y autonomía pedagógica, a través del estudio de lo recogido en trabajos teóricos, normativa y legislación, exposición de experiencias institucionales y trabajos de investigación previos.

\subsection{La cuestión en el sistema universitario español}

El análisis del sistema universitario español conforme a la autonomía, después de su entrada en el proceso Bolonia (EEES, 1999), ha ido reformando su estructura según los estándares europeos. El programa estatal de indicadores de la educación para 2000-2020 reconoce muchos de estos problemas y establece objetivos específicos y tareas para su implementación.

Desde la LOU (2001) se mencionan las funciones y autonomía que tienen las Universidades y concretamente se habla de la autonomía universitaria y proponen distintas actuaciones entre las que destacan la elaboración de sus propias normas de organización y funcionamiento; creación de estructuras para dar cabida a

la investigación y docencia; elaboración y aprobación de planes de estudio e investigación y de enseñanzas especificas de formación a lo largo de toda la vida; selección, formación y promoción del personal docente e investigador y de administración y servicios, así como la determinación de las condiciones en que han de desarrollar sus actividades. (p. 14)

Se trata de que docentes, investigadores y estudiantes cumplan con sus funciones y responsabilidades, teniendo en cuenta las necesidades educativas que puedan presentar y dar respuesta a todo tipo de alumnado, construyendo un aprendizaje reflexivo-crítico que ayude al desarrollo integral del estudiante.

En cuanto al desarrollo normativo en materia de inclusión en la educación superior en España, lo cierto es que no existe un marco legal coherente y robusto. En la LOU se expone que las Universidades deben garantizar la igualdad de oportunidades, eliminar cualquier forma de discriminación y establecer medidas de acción positiva para garantizar la inclusión, pero, sin embargo, no se acoge en su totalidad a los preceptos de la Convención (Naciones Unidas, 2006) ni se desarrolla de forma homogénea entre las Universidades, lo que supone situaciones de discriminación.

Si se relacionan estas cuestiones con las investigaciones previas sobre la materia, se encuentran los siguientes resultados que se detallan a continuación y que ofrecen información concreta sobre el estado de la cuestión en España.

En primer lugar, la percepción general de los estudiantes acerca de la institución universitaria es adecuada, incluyendo aquellos aspectos relacionados al carácter inclusivo que promueve (Serrano y Capdevila, 2013). Concretamente, en la percepción de los estudiantes con discapacidad, éstos identifican déficits en la normativa que no ofrece garantías para una educación de calidad y la relevancia de los servicios de apoyo, orientación y asesoramiento entre otros (Fernández, 2017).

Por otro lado, la investigación de Molina Béjar (2010) señala que, a pesar de la existencia de un marco normativo que garantiza la inclusión educativa, el desinterés y abandono del tema en la educación superior es relevante. Asimismo, plantea el desarrollo de una cultura de la diversidad. Esto implica asumir que los sistemas educativos deben ofrecer una respuesta transversal de carácter inclusivo acorde al desarrollo de una sociedad más justa y equitativa para promover una educación de calidad (Arizabaleta Domínguez y Ochoa Cubillos, 2016; Jaimes et al., 2009). 
En contraste a esta cuestión, Olivencia y Gerdel (2017) consideran que la educación superior universitaria ha realizado determinados cambios y avances en pro de la inclusión a pesar de su carácter más teórico que práctico. Una idea que apostillan Olivencia y Hernández (2012) y añaden su apuesta por ofrecer discursos novedosos y prácticas docentes propias de la inclusión como seña de calidad y excelencia de las Universidades.

Según los últimos datos publicados en la "Guía de atención a la discapacidad en la Universidad 20192020" (Fundación Universia, 2020) en este curso un total de 22.818 universitarios con discapacidad, estudian en las Universidades españolas mostrando estos datos una ligera tendencia positiva. No obstante, la dotación de medidas para garantizar el derecho a la educación inclusiva de todos los estudiantes en la distintas universidades españolas es desigual y muestra carencias en aspectos como los siguientes que se destacan: planes de formación en materia de discapacidad para el profesorado (Medina-García et al., 2020); asesoramiento específico a personas con enfermedad mental; programas de atención a estudiantes con altas capacidades y enseñanzas de especial atención a la discapacidad (Fundación Universia, 2020).

Estos datos vienen a hacer patente una de las premisas de este trabajo en torno a los déficits en materia de autonomía para garantizar la inclusión en el ámbito universitario, motivada entre otras cuestiones por la falta de formación ofrecida al profesorado sobre la materia, a pesar de las necesidades y demandas del profesorado al respecto (Medina-García et al., 2020).

\subsection{El panorama universitario en Colombia}

$\mathrm{Al}$ analizar el contexto latinoamericano, y en concreto el colombiano, se visibilizan grandes avances en materia de legislación, políticas y normativa para el sector de la educación de diferentes países en lo relacionado con la educación inclusiva, el enfoque de derechos y las acciones a desarrollar en favor de los grupos poblacionales en situaciones de dificultad social, aunque con dificultades para materializar la legislación en políticas educativas y en la práctica pedagógica (Fajardo, 2017; Terigi, 2014).

Colombia, ha sido uno de esos ejemplos con avances importantes que giran en relación al desarrollo de la educación inclusiva: "el planteamiento de una política pública nacional, un conjunto de las normativas que la soportan y los fundamentos jurisprudenciales trazados por la Corte Constitucional que orientan las prácticas inclusivas desarrolladas por los entes territoriales e institucionales” (Fajardo, 2017, p. 184). En el año 2013 el Ministerio de Educación Nacional de Colombia (en adelante MEN) presenta los "Lineamientos de Educación Superior Inclusiva”. El objetivo de este documento es acompañar y orientar a las instituciones de educación superior, en quienes recae la función de desarrollar políticas institucionales que favorezcan el acceso, permanencia y graduación especialmente de aquellos estudiantes más proclives a ser excluidos del sistema educativo (MEN, 2013, p. 5). Estos lineamientos adoptan como principios fundamentales la integralidad y la flexibilidad, así como de diversidad, calidad, participación, equidad, interculturalidad y pertinencia; los cuales son fundamentados a partir de los aportes de autores como de Mel Ainscow, Artemi Sakellariadis y Clementina Acedo.

De acuerdo con la Universidad Nacional Abierta y a Distancia del mismo país (UNAD, 2014), la educación inclusiva abarca un estudio detallado y sistemático de las diferentes discriminaciones que se pueden presentar a nivel institucional y/o en un sistema educativo para, desde un enfoque inclusivo, proponer estrategias que respondan a las particularidades y condiciones diversas de los estudiantes. La educación inclusiva se entiende desde parámetros de diversidad poblacional definidas por el contexto al cual pertenecen, sus historias de vida, su situación sociofamiliar, étnica, económica, además sus necesidades y potencialidades en su proceso de aprendizaje. Desde esta perspectiva, la educación superior inclusiva en Colombia trasciende la integración y la permanencia en el sistema educativo de aquellas personas en situaciones de riesgo de exclusión social (MEN, 2013).

El panorama de la educación superior en Colombia está marcado por su, mayoritariamente, carácter privado y tercerizado, lo cual dificulta el alcance y mantenimiento de la calidad educativa, la transparencia y la medición de las prácticas desarrolladas (Fajardo, 2017), dejando a la voluntad de las instituciones educativas el desarrollo de las distintas iniciativas a favor de la inclusión.

Para Arizabaleta y Ochoa (2016), 
toda institución de educación superior colombiana, pública o privada, necesita un equipo líder que intérprete y comprenda el concepto de educación superior inclusiva en su vertiente de docencia, investigación, proyección social, bienestar institucional y su estructura y gestión administrativa y financiera; sin que dé pie a errores conceptuales al confundir la educación inclusiva con inclusión social, inclusión educativa, integración, necesidades educativas. (p. 44)

Por ejemplo, Garzón y Molina (2014), ponen de relieve el programa IncluSer de la Universidad del Rosario (Colombia) a partir del cual se han dado una serie de desarrollos institucionales especialmente en la autonomía tanto académica como social de los estudiantes con discapacidad y su participación en la vida universitaria.

Se reconoce, por tanto, la preocupación de estas instituciones por posicionarse como universidades incluyentes, sin embargo, las atenciones que ofrecen para lograr cumplir con sus políticas institucionales continúan siendo individualizadas e integradoras, ya que no profundizan en el diseño de estrategias para saber abordar el tema (UNAD, 2014).

Principalmente, las acciones que se han desarrollado son "medidas para facilitar el acceso, disponer de programas o servicios de apoyo para la permanencia e incorporar la temática en los proyectos de desarrollo institucional, de investigación y de proyección social; todo ello en un intento por llevar a la práctica la educación inclusiva e incluyente” (Fajardo, 2017, p. 195). Pero, además, se requiere un desarrollo de la autonomía institucional y liderazgo que apunte a tres retos clave: sensibilización de la comunidad educativa, la elaboración de un instrumento y recogida de datos que nos permita conocer el estado de educación superior inclusiva en Colombia y el planteamiento y puesta en práctica de una política de educación superior inclusiva conforme a los elementos que caracterizan los lineamientos de acreditación del Consejo Nacional de Acreditación de instituciones educativas (Arizabaleta y Ochoa, 2016).

\section{Síntesis de los hallazgos}

Fruto de la revisión teórica realizada sobre el tema se ha aportado la exposición de los elementos revisados y una serie de reflexiones que permiten valorar el panorama actual sobre la educación superior inclusiva y la autonomía pedagógica sustentados sobre los principales hallazgos.

a) La autonomía pedagógica es clave para el desarrollo de una educación inclusiva, pero requiere una estructura única y común para el desarrollo de la educación superior inclusiva

Tras el desarrollo de nuestro trabajo se muestra la necesidad que existe de la implementación en la educación superior de herramientas y estrategias que ayuden a fomentar una educación inclusiva de calidad teniendo en cuenta la autonomía pedagógica como elemento fundamental. Se considera la autonomía pedagógica clave para el desarrollo de una educación inclusiva y al respecto Casanova (2020) expone que según la autonomía que apliquen las instituciones educativas así será el grado de inclusión. Se ha podido comprobar cómo en las enseñanzas medias la autonomía pedagógica de los centros trabaja tímidamente la inclusión, sin que esto tenga continuidad en otras etapas educativas como es la universitaria, en la que su desarrollo muestra mucha variabilidad y carencias según el contexto. Se valora como una de las causas de esta situación la falta de obligatoriedad normativa y la creación de marcos estructurales que canalicen y faciliten las acciones en pro de la educación superior inclusiva. Por ejemplo, en ambas realidades estudiadas se produce una desconexión en el trasvase de información entre las instituciones de niveles educativos previos y la universitaria que permitan un conocimiento sobre las situaciones previas educativas vividas y los procesos iniciados en niveles educativos anteriores.

\section{b) Desconexión entre el imperativo legal y la implementación práctica}

Existe una gran desconexión entre los preceptos legales y la aplicación práctica de los mismos. En el territorio colombiano, de forma específica, a pesar de los esfuerzos por las aclaraciones por crear un marco de referencia único, la multiplicidad de experiencias provoca disparidad de interpretaciones. Se requiere una aplicabilidad clara y mediciones nacionales únicas para asegurar una uniformidad en el alcance de derechos, sin que ello interceda en la autonomía pedagógica e institucional de las instituciones de educación superior. En el caso de España, la situación es similar. La desconexión que existe entre los postu- 
lados normativos y la aplicación real es la mayor de las dificultades para lograr la implementación de los aspectos referidos a la autonomía pedagógica y la inclusión.

c) Las carencias y obstáculos en el ejercicio de la autonomía universitaria impactan en la capacidad de las instituciones de garantizar la inclusión

A pesar de la apuesta de la comunidad europea por la autonomía universitaria, la realidad o aplicación práctica es otra, valorándose como deficiente y dispar, dificultando el posible desarrollo de herramientas para dar respuesta a la diversidad desde una visión de inclusión. Sin embargo, a pesar de ser necesario, la presencia de altos niveles de autonomía no es determinante para el afianzamiento de una educación superior inclusiva. Muestra de ello, las universidades colombianas, de forma concreta, gozan de altos niveles de autonomía, pero ese hecho no siempre deriva en una trayectoria institucional en pro de los derechos y responsabilidad social. A pesar de los esfuerzos por crear un sistema de acreditación y evaluación a nivel nacional es una valoración que se realiza a posteriori y bajo el cual caben multitud de modelos universitarios, en los cuáles los enfoques sobre inclusión son también variados.

\section{d) La autonomía pedagógica y la inclusión: entre la responsabilidad social y la calidad}

Se establece la autonomía pedagógica y la inclusión como un elemento de calidad educativa (Chiroleu, 2009; Estermann, 2015). La autonomía pedagógica es clave para la viabilidad de la educación superior inclusiva si se asume que ésta debe dar respuestas pertinentes a contextos concretos, asentados en buenos diagnósticos; sin que ello suponga el establecimiento de prácticas individualizadas que serían más propias del enfoque integrador e incluso del modelo médico-rehabilitador.

La responsabilidad social se vincula con el concepto de autonomía pedagógica, pues la educación superior debe construir propuestas de organización que busquen mejores formas de atender a la diversidad de la población (Unesco, 2000) y para ello se debe "avanzar hacia diseños universales en los que el currículo y la enseñanza consideren de entrada la diversidad de los estudiantes" (Blanco, 2008, p. 8). Y es que, una Universidad que acoge e implementa los fundamentos de la inclusión es una mejor Universidad y tiene como eje central la calidad de la enseñanza pensada para todas las personas (Ainscow, 2001).

Además, al focalizar el análisis en las dos realidades que se presentan en este trabajo se observa la necesidad de proceder a una mejora de los indicadores de calidad en relación a autonomía e inclusión en el ámbito universitario. En España, no existe una apuesta firme para su consolidación en la educación superior, con respecto a la autonomía e inclusión, claves para el desarrollo social sostenible al que está comprometido la Universidad. En Colombia el propósito final no queda claro: los Lineamientos de Educación Superior Inclusiva (MEN, 2013) se han entendido como una manera de asegurar la calidad educativa y los derechos dentro de la propia institución, pero todavía queda camino por recorrer para comprender que dicha acción implica también un ejercicio de extensión universitaria en lo que se entiende como responsabilidad social.

En cualquier caso, no debería existir ninguna tensión entre la autonomía universitaria (en cualquiera de sus formas, y en concreto en su dimensión pedagógica), en aras de asegurar buenos niveles de calidad, y la consolidación de la educación superior inclusiva y su deber respecto a la responsabilidad social.

\section{e) La implantación de un diseño universal como parte del ejercicio de la autonomía pedagógica}

El diseño universal se entiende como el acogimiento de perspectivas abiertas e inclusivas esenciales para promover la implicación de los estudiantes con discapacidad en la Universidad (López-Bastias et al., 2020). Este reto se ve dificultado por el ejercicio de la autonomía pedagógica de cada Universidad y por la propia configuración de los grados, su contenido "impuesto" y preestablecido a pesar lo expuesto en el Real Decreto 1393/2007 de 29 de octubre, por el que se establece la ordenación de las enseñanzas universitarias oficiales en su Principio 5 y describe los principios generales que deberán inspirar el diseño de los títulos universitarios y currículos académicos desde el respeto y promoción de los Derechos Humanos y los principios de accesibilidad universal y diseño para todos (Villoria y Fuentes, 2015).

\section{f) La formación del profesorado como palanca de cambios en la educación superior}

Para concluir, se necesita de instituciones que se comprometan con la mejora de la calidad y equidad en educación superior reconozcan que los docentes son quienes tienen la capacidad de implementar en sus aulas mecanismos inclusivos e ir adaptándose a la realidad educativa que se presente. De tal modo, la for- 
mación del profesorado se establece como un aspecto fundamental para promover cambios en términos de autonomía que permitan el afianzamiento de una educación superior inclusiva (Almenta y Muñoz, 2007; Olivencia y Hernández, 2012) y para el desarrollo integral del alumno en todas sus vertientes. La oferta de formación del personal docente investigador es una cuestión que se establece según prioridades institucionales y que rara vez vienen impuestas, por lo tanto, es parte del ejercicio de su autonomía y reflejo de sus prioridades y compromiso y responsabilidad social el poder diseñar unos programas de formación en relación a la educación inclusiva para que tengan las estrategias necesarias para dar respuesta a la diversidad en el alumnado universitario (Medina-García et al., 2020).

\section{g) El reto iberoamericano: garantizar y alcanzar niveles similares de inclusión en las instituciones educativas superiores independientemente del contexto}

La revisión de la situación del país de las realidades universitarias española y colombiana ha permitido analizar los detalles de dos maneras diferentes de abordaje del tema, difícilmente equiparables ya que existen diferencias sustanciales en la propia estructura de la concepción del sistema universitario y de lo que significa la educación superior inclusiva. En Colombia, se ha planteado como una apuesta que nace del Estado, pero cuyo cumplimiento o afianzamiento recae directamente sobre la autonomía de las diferentes instituciones de educación superior del país. Como ya se ha mencionado, las Universidades colombianas gozan de altos niveles de autonomía, pero en ocasiones parece que quede a merced de su voluntariedad, su postura política y su cultura institucional el desarrollo de determinadas prioridades o acciones. Fruto de esa coyuntura nacen prácticas de difícil valoración, seguimiento y medición. En cambio, en España, tanto la autonomía universitaria como la necesidad de instaurar una educación superior inclusiva se encuentran presentes en la normativa y se plantean como cambios necesarios y demandas pendientes de cumplir por parte de los organismos europeos, pero la implementación de estas cuestiones se realiza de forma laxa cubriendo en la mayoría de los casos aspectos concretos y poco profundos relacionados con el derecho de acceso, la garantía de apoyos y niveles muy básicos de accesibilidad y adaptaciones curriculares, quedando pendiente retos como la accesibilidad universal y garantizar el diseño universal para todas las personas.

\section{Conclusiones}

Retomando el objetivo propuesto, y a modo de conclusión, se hace patente la relación existente entre la educación inclusiva y la autonomía pedagógica a nivel universitario, retroalimentandose ambas, siendo ineludible para la potencialización de los sistemas educativos tanto español como colombiano, en una apuesta afianzar y garantizar derechos, y mantener niveles de excelencia educativa. En dicha relación, el instaurar una educación inclusiva a nivel de estudios superiores requiere de niveles altos de autonomía, a la vez que, la autonomía institucional debe regirse por una apuesta hacia la promoción de valores y procesos propios de la inclusión.

En el desarrollo de este trabajo se ha encontrado como gran obstáculo que apenas se localizan trabajos o investigaciones previas que aborden y profundicen sobre esta cuestión. No obstante, con el análisis realizado en la bibliografía, así como de la comparativa ofrecida de los contextos iberoamericanos, se pretende generar elementos iniciales para el planteamiento del tema, sobre todo una base conceptual y teórica. En definitiva, de todo lo expuesto, deriva la novedad de este trabajo, el cual supone importantes implicaciones para las instituciones de educación superior iberoamericanas, tanto para la gestión como para las políticas educativas pues ofrece evidencias e información relevante sobre cómo promover la inclusión en el ámbito universitario a través de la gestión de la autonomía, aspecto innovador no contemplado antes en la literatura. Otro inconveniente detectado es la notable diferencia presente en la conceptualización de la autonomía en función de la etapa educativa en incluso del país, lo que supone una dificultad a la hora de extrapolar o transferir los aspectos más relevantes y los hallazgos descubiertos.

En este sentido es importante destacar que, como consecuencia del mismo, se perfilan claras líneas de investigación futuras centradas en la imperiosa necesidad de realizar trabajos de investigación y empíricos que permitan sentar bases del tema; sobre todo, además de refuerzo a nivel teórico, urgen estudios alrededor de la práctica, estudios de percepción, exposición y valoración de metodologías, resultados de experiencias, propuestas de evaluación etc. 


\section{Referencias}

Aghion, P., Dewatripont, M., Hoxby, C., Mas-Colell, A. y Sapir, A. (2008). Higher aspirations: An agenda for reforming european universities. Bruegel Blueprint Series.

Ainscow, M. (2001). Desarrollo de escuelas inclusivas: Ideas, propuestas y experiencias para mejorar las instituciones escolares. Narcea.

Ainscow, M. y West, M. (2008). Mejorar las escuelas urbanas. Narcea.

Ainscow, M. y Miles, S. (2008). Por una educación para todos que sea inclusiva: ¿Hacia dónde vamos ahora? Perspectivas, 38(1), 17-44.

Alcaín, E. y Medina-García, M. (2017). Hacia una educación universitaria inclusiva: Realidad y retos. Revista Digital de Investigación en Docencia Universitaria, 11(1), 4-19. https://doi.org/10.19083/ridu.11.530

Almenta, E. y Muñoz, J. (2007). ¿Estamos formados para trabajar en una escuela inclusiva? En VVAA, Actas del V Congreso Internacional Educación y Sociedad. La educación, retos del siglo XXI (pp. 145-149). Codoli.

Arizabaleta, S. L. y Ochoa, A. F. (2016). Hacia una educación superior inclusiva en Colombia. Pedagogía y Saberes, 45, 41-52. https://doi.org/10.17227/01212494.45pys41.52

Asociación Europea de Universidades. (2010). Declaración de Lisboa. https://eua.eu/resources/publications/624:es-declaración-de-lisboa.html

Ávalos, B. (1994). Creatividad versus autonomía profesional del profesor. Consideraciones sobre el tema, derivadas de la investigación pedagógica. Pensamiento Educativo, 14(1), 13-48.

Ávila, J., Contreras, M. y Gardea, J. (2019). Autonomía curricular: un desafío en la práctica docente. En SEP. (Ed.), Actas del III Congreso Nacional de investigación sobre educación normal. SEP.

Blanco, R. (2008). Marco conceptual sobre educación inclusiva. Confinted, 48(2), 5-14.

Bolívar, A. (2010a). El liderazgo educativo y su papel en la mejora: Una revisión actual de sus posibilidades y limitaciones. Psicoperspectivas, 9(2), 9-33. https://doi.org/10.5027/psicoperspectivas-Vol9-Issue2-fulltext-112

Bolívar, A. (2010b). ¿Cómo un liderazgo pedagógico y distribuido mejora los logros académicos? Revisión de la investigación y propuesta. Magis, Revista Internacional de Investigación en Educación, 3(5), 79-106.

Bolívar, A. (2018). La inspección educativa en un marco de autonomía escolar, una inevitable reestructuración. Fórum de Aragón, 24, 10-19.

Bolívar, A. (2019). Una dirección escolar con capacidad de liderazgo pedagógico. La Muralla.

Carrillo, S. y Flores, I. (2013). The formative and transformer value of cooperation. An experience of educational practices in Guatemala. Revista Iberoamericana de Educación, 61, 121-141. https://doi.org/10.35362/rie610604

Casanova, M. A. (2012). El diseño curricular como factor de calidad educativa. REICE. Revista Iberoamericana sobre Calidad, Eficacia y Cambio en Educación, 10(4), 6-20.

Casanova, M. A. (2016). Diseño curricular para la educación inclusiva. En I. E. Ramírez (Ed.), Voces de la inclusión: Interpelaciones y críticas a la idea de inclusión escolar (pp. 90-108). Praxis editorial

Casanova, M. A. (2020). Miradas de futuro: Educación inclusiva para la sociedad democrática. Avances en Supervisión Educativa, 33, 68-83.

Chiroleu, A. (2009). Políticas públicas de inclusión en la educación superior los casos de Argentina y Brasil. Pro-Posições, 20(2), 141-166. https://doi.org/10.1590/S0103-73072009000200010

Christensen, T. (2011). University governance reforms: Potential problems of more autonomy? Higher Education, 62(4), 503-517. https://doi.org/10.1007/s10734-010-9401-z

Comisión Europea. (2006). Cumplir con la agenda de modernización de las universidades: Educación, investigación e innovación. Comisión Europea. 
Day, C., Gu, Q. y Sammons, P. (2016). The impact of leadership on student outcomes: How successful school leaders use transformational and instructional strategies to make a difference. Educational Administration Quarterly, 52 (2), 221-258. https://doi.org/10.1177/0013161X15616863

De Boer, H., Jongbloed, B., Enders, J. y File, J. (2010). Progress in higher education reform across Europe: Governance reform. Comisión Europea.

Desimone, P. (2007). Análisis de las prácticas pedagógicas y su efecto en la calidad de los ambientes de aprendizajes de los párvulos, en el $2^{\circ}$ nivel de transición de la educación parvularia en la comuna de Curicó, VII Región, Chile. REICE. Revista Iberoamericana sobre Calidad, Eficacia y Cambio en Educación, 5, 36-47

Diamond, J. y Spillane, J. (2016). School leadership and management from a distributed perspective: A 2016 retrospective and prospective. Management in Education 30(4),147-154. https://doi.org/10.1177/0892020616665938

EEES. (1999). Espacio europeo de educación superior. EEES.

Estermann, T. (2015). University autonomy in Europe. University Education, 3, 28-32.

Estermann, T. y Bennetot, E. (2011). Financially sustainable universities II: European universities diversifying income streams. European University Association.

Estermann, T., Nokkala, T. y Steinel, M. (2011). University autonomy in Europe II. The scorecard. European University Association.

Fajardo, M. S. (2017). La educación superior inclusiva en algunos países de Latinoamérica: Avances, obstáculos y retos. Revista Latinoamericana de Educación Inclusiva, 11(1), 171-197. https://doi.org/10.4067/s0718-73782017000100011

Fedulova, I., Ivanova, V., Atyukova, O. y Nosov, V. (2019). La educación inclusiva como base para el desarrollo sostenible de la sociedad. Revista de Investigación en Educación en Estudios Sociales, 10(3), 118-135.

Fernández Vivas, Y. (2017). La libertad de cátedra: Concepto, límites y armonización con otros derechos y obligaciones. CEDU.

Fernández, A. C. (2017). Educación inclusiva en las instituciones de educación superior: Narrativas de estudiantes con discapacidad. Revista Española de Discapacidad, 5(1), 43-61. https://doi.org/10.5569/2340-5104.05.01.03

Fullan, M. (2011). Choosing the wrong drivers for whole system reform. Centre for Strategic Education. https://doi.org/10.17323/1814-9545-2011-4-79-105

Fumasoli, T., Gornitzka, A. y Maassen, P. (2014). University autonomy and organizational change dynamics. ARENA.

Fundación Universia. (2020). Guía de atención a la discapacidad en la Universidad 2019-2020. Universia.

Garces, P. y Letelier, M. (2016). Description of the graduate students intellectual and etiology profile, of a university inclusion program for young people with mild mental disabilities. Revista de Educación Inclusiva, 9(2), 2012015.

García, M. M. (2020). La universidad inclusiva: Un reto de nuestro sistema educativo para garantizar el derecho a la educación. En L. Ortiz y J. J. Carrión (Ed.), Educación inclusiva: Abriendo puertas al futuro (pp. 121-137). Universidad de Almería. https://doi.org/10.2307/j.ctv153k3m3.9

Garzón, D. K. y Molina, B. K. (2014). IncluSer. Del apoyo, a la participación y reconocimiento de estudiantes con discapacidad en la Universidad del Rosario. Editorial Universidad del Rosario.

Gómez, Y. y García-Vita, M. M. (2017). Hacia una educación superior inclusiva. ReiDoCrea, 6, 300-319.

González, I. y De León, C. (2011). The webquest invadiv: A proposal to promote educational cooperative work in the classroom. RIED-Revista Iberoamericana de Educacion a Distancia, 14(2), 167-188.

González, O., Fontaneda, I., Camino, M. y Revilla, A. (2015). La responsabilidad social en las universidades españolas 2014/15. Instituto Universitario de Análissi Económico y Social.

Jaimes, G. I. B., Delgado, M. F. B. y Pineda, D. R. V. (2009). Discapacidad en Colombia: Un reto en la educación superior inclusiva. Revista Colombiana de Rehabilitación, 41, 37-53. 
Jiménez Delgado, M., Jareno, D. y Draoui, B. (2016). Educational and social inclusion of the young women from immigrant origin during post-compulsory education. Prisma Social, 16, 411-449.

Jongbloed, B., De Boer, H., Enders, J. y File, J. (2010). Progress in higher education reform across Europe: Funding reform. Executive summary and main report. Comisión Europea.

Kerr, C. (1993). Universal issues in the development of higher education. En J. B. Balderston y F. E. Balderston (Eds.), Higher education in Indonesia: Evolution and reform (pp.19-35). Center for Studies in Higher Education.

Kerr, C. (2001). The uses of the university. Harvard University Press.

Kezar, A. y Eckel, P. D. (2004). Meeting today's governance challenges: A synthesis of the literature and examination of a future agenda for scholarship. Journal of Higher Education, 75(4), 371-399.

https://doi.org/10.1080/00221546.2004.11772264

Leithwood, K., Day, C., Sammons, P., Harris, A. y Hopkins, D. (2006). Successful school leadership. What it is and how it influences pupil learning. National College for School Leadership/University of Nottingham

Llorent, V. J., Zych, I. y Varo-Millán, J. C. (2020). University academic personnel's vision of inclusive education in spanish universities. Culture and Education, 32(1), 147-181. https://doi.org/10.1080/11356405.2019.1705593

López, L., Etxabe, E., y Montero, D. (2016). Views of students with disabilities about their experience in secondary education in The Basque country, Spain. Journal of Research in Special Educational Needs, 16, 1090-1094. https://doi.org/10.1111/1471-3802.12254

López-Bastias, J. L., Moreno-Rodriguez, R. y Díaz-Vega, M. (2020). Attention to the special educational needs of university students with disabilities: The CAUSSEN tool as part of the educational inclusion process. Culture and Education, 32(1), 27-42. https://doi.org/10.1080/11356405.2019.1705563

Louis, K.-S. y Wahlstrom, K. (2011). Shared and instructional leadership. When principals and teachers successfully lead together. En K. Leithwood y K.-S. Louis (Eds.), Linking leadership to student learning (pp. 25-41). Jossey Bass.

Maasen, P. (2008). The modernization of european higher education: National policy dynamics. En A. Amral, I. Bleiklie, y C. Musselin (Eds.), From governance to identity. A Festschrift for Mary Henkel, (pp. 95-112). Springer. https://doi.org/10.1007/978-1-4020-8994-7_8

Martínez, E. A. (2017). La responsabilidad social universitaria y la discapacidad: Una contribución a lo que la universidad tiene que ser además. Anales de Derecho y Discapacidad, 2, 107-123.

Martínez-Valdivia, E., García-Martínez, I. e Higueras-Rodríguez, L. (2018). El liderazgo para la mejora escolar y la justicia social. Un estudio de caso sobre un centro de educación secundaria obligatoria. REICE. Revista Iberoamericana sobre Calidad, Eficacia y Cambio en Educación, 16(1), 35-51.

https://doi.org/10.15366/reice2018.16.1.003

Medina-García, M. (2017). La educación inclusiva como mecanismo de garantía de la igualdad de oportunidades y no discriminación de las personas con discapacidad. Una propuesta de estrategias pedagógicas inclusivas. Ediciones Cinca.

Medina-García, M., García-Vita, M. M. e Higueras-Rodríguez, L. (2020, 7 de octubre). La formación del profesorado universitario en discapacidad y atención a la diversidad [Comunicación]. CUICIID 2020, Congreso Universitario internacional sobre contenidos, investigación, innovación y docencia, Barcelona, España.

Mesa, R., Consuelo, M. y Tabares Idárraga, L. E. (2000). Métodos de investigación en educación. Revista de Ciencias Humanas, 21, 57-76.

Ministerio de Educación Nacional. (2013). Lineamientos de politica de educación superior inclusiva. Ministerio de Educación Nacional.

Molina Béjar, R. (2010). Educación superior para estudiantes con discapacidad. Revista de Investigación, 34(70), 109128

Moral, C., Higueras-Rodríguez, L., Martín-Romera, A., Martínez-Valdivia, E. y Morales-Ocaña, A. (2020). Effective practices in leadership for social justice. Evolution of successful secondary school principalship in disadvan- 
taged contexts. International Journal of Leadership in Education, 23(2), 107-130.

https://doi.org/10.1080/13603124.2018.1562100

Moriña, A., Cortés, M. D. y Melero, N. (2014). ¿Currículos inclusivos en la educación superior española? Los estudiantes con discapacidades se expresan. Discapacidad y Sociedad, 29(1), 44-57.

Naciones Unidas. (1948). Declaración universal de los derechos humanos. Naciones Unidas.

Naciones Unidas. (2006). Convención internacional sobre los derechos de las personas con discapacidad. Naciones Unidas.

Ng, P. T. (2011). How the world's most improved school systems keep getting better. Journal of Educational Change, 12, 463-477. https://doi.org/10.1007/s10833-011-9174-x

Nielsen, T. (2010). Lost in translation? Rethinking first nation education via LUCID insights. International Review of Education, 56(4), 411-433. https://doi.org/10.1007/s11159-010-9168-6

Niemi, H., Lavonen, J., Kallioniemi, A. y Toom, A. (2018). The role of teachers in the Finnish educational system: High professional autonomy and responsibility. En A. Toom y H. Niemi (Eds.), The teacher's role in the changing globalizing world (pp. 47-61). Brill Sense. https://doi.org/10.1163/9789004372573_004

Núñez, M. y Alonso, I. (2009). La responsabilidad social en el mapa estratégico de las universidades públicas. Pecvnia, 9, 157-180. https://doi.org/10.18002/pec.v0i9.666

Observatorio Estatal de la Discapacidad. (2019). Observatorio estatal de la discapacidad._https://www.observatoriodeladiscapacidad.info/

Ocampo, A. (2013). Inclusión, universidad y discapacidad: Una complejidad epistémica más allá de la igualdad de oportunidades. Temas de Educación, 19(2), 89-103.

Olivencia, J. y Gerdel, M. (2017). La educación inclusiva como constructo pedagógico en el alumnado universitario de educación primaria. Revista de Educación Inclusiva, 8(2), art. 6.

Olivencia, J. y Hernández, A. (2012). La educación inclusiva en la universidad del siglo XXI. Un proceso permanente de cambio. Revista Electrónica de Investigación y Docencia, 8, 41-62.

Olsen, J. P. (2007). The institutional dynamics of the european university. En P. Maassen y J. P. Olsen (Eds.), University dynamics and european integration (pp. 25-55). Springer. https://doi.org/10.1007/978-1-4020-5971-1_2

Paradeise, C., Bleiklie, I., Enders, J., Goastellec, G., Michelsen, S. y Reale, E. (2009a). Reform policies and change processes in Europe. En J. Huisman (Ed.), International perspectives on the governance of higher education. Alternative frameworks for coordination (pp.88-106). Routledge.

Paradeise, C., Reale, E. y Goastellec, G. (2009b). A comparative approach to higher education reforms in Western Europe Countries. En C. Paradeise, E. Reale, I. Bleiklie y E. Ferlie (Eds.), University governance. Western European comparative perspectives (pp. 197-225). Springer. https://doi.org/10.1007/978-1-4020-9515-3_9

Parcerisa, L. (2016). Nueva gestión pública y reforma educativa: La recontextualización de la autonomía escolar en diferentes contextos escolares en Cataluña. Revista Portuguesa de Educação, 29(2), 359-390.

https://doi.org/10.21814/rpe.7928

Pérez Gómez, A. I. (1998). La cultura escolar en la sociedad neoliberal. Morata

Reichert, S. y Tauch, C. (2005). Trends IV: European universities implementing Bologna. EUA.

Robinson, V., Hohepa, M. y Lloyd, C. (2009). School leadership and student outcomes: Identifying what works and why. Best evidence synthesis iteration. Ministry of Education.

Rodríguez, J., Rodríguez, A. J., Artíles, J., Aguiar, M. V. y Alemán, J. A. (2013). El acceso a la dirección escolar: dificultades y necesidades. Educar, 49(1), 105-125. https://doi.org/10.5565/rev/educar.13

Salinas, D. (1994). La planificación de la enseñanza: ¿Técnica, sentido común o saber profesional? Teoría y desarrollo del currículum. En J. F. Ángulo y N. Blanco (Eds.), Teoría y desarrollo del currículum (pp. 35-160). Ediciones Aljibe.

Sarasúa, A. (2013). La dirección escolar, lunes y sombras. Padres y Maestros, 350, 41-44. 
Segura, J., Gairín, J. y Silva, P. (2020). Implicaciones de la inspección educativa en Cataluña en el proceso de autonomía de centros. REICE. Revista Iberoamericana sobre Calidad, Eficacia y Cambio en Educación, 19(1), 61-82. https://doi.org/10.15366/reice2021.19.1.004

Serrano, G. y Capdevila, M. (2013). Diversidad cultural y ciudadanía. Hacia una educación superior inclusiva. Educación XX1, 16(1), 57-81.

Silva, P., Del-Arco, I. y Flores, O. (2018). La formación de directores escolares en Cataluña. Lecciones aprendidas a cinco años del decreto de dirección. Bordón, 70(1),109-124.

Stainback, S. y Stainback, W. (1999). Aulas inclusivas. NARCEA.

Stenhouse, L. (1998). Investigación y desarrollo del curriculum. Ediciones Morata.

Strauss, A. y J. Corbin. (1990). Basics of qualitative research: Grounded theory procedures and techniques. Sage Publications.

Sursock, A., Smidt, H. y Davies, H. (2010). Trends 2010: A decade of change in european higher education. European University Association.

Terigi, F. (2014). Trayectorias escolares e inclusión educativa. Del enfoque individual al desafío para las políticas educativas. En Marchesi A., Blanco R. y Hernández L. (Coord.), Avances y desafíos de la educación inclusiva en Iberoamérica. Organización para los estados Iberoamericanos. Metas.

Torres, H. A. (2018). La autonomía curricular exige capacidad reflexiva y constructiva de docentes. Revista de Evaluación para Docentes y Directivos, 2(4), 67-88.

Universidad Nacional Abierta y a Distancia. (2014). Educación inclusiva en la educación superior en Colombia y en la universidad nacional abierta y a distancia. Estado del Arte. UNAD.

Unesco. (1998). Declaración mundial sobre la educación superior en el siglo XXI: Visión y scción. Unesco.

Unesco. (2000). Marco de acción de Dakar. Educación para todos: Cumplir nuestros compromisos comunes. Unesco.

Vega, N. R. (2009). Necesidades emergentes y responsabilidad social universitaria. Revista Alternativas. Cuadernos de Trabajo Social, 16, 65-76. https://doi.org/10.14198/ALTERN2009.16.5

Vilaverde, E. (2020). Online instruction. A system for educational inclusion in the university setting. Culture and Education, 32(1), 106-122. https://doi.org/10.1080/11356405.2019.1705561

Villoria, E. D. y Fuentes, S. S. (2015). Diseño universal para el aprendizaje como metodología docente para atender a la diversidad en la universidad. Aula Abierta, 43(2), 87-93. https://doi.org/10.1016/j.aula.2014.12.002

\section{Breve CV de las autoras}

\section{Marta Medina-García}

Doctora en Pedagogía. Profesora en la Universidad de Jaén. Departamento de Pedagogía, del área de Didáctica y Organización Escolar. Miembro del Grupo de Investigación HUM782 - Diversidad, Discapacidad y Necesidades Educativas Especiales. Las líneas de investigación son fundamentalmente la educación inclusiva, atención a la diversidad y discapacidad, la formación docente y las metodologías activas. Coordinadora de proyectos de inclusión a nivel nacional e internacional. Coordinadora y docente en el Máster Propio en Discapacidad, Autonomía Personal y Atención a la Dependencia.

Email: mameding@ujaen.es

ORCID ID: https://orcid.org/0000-0003-0520-3635

\section{Lina Higueras-Rodríguez}

Profesora del área de Didáctica y Organización Escolar del departamento de Educación de la Universidad de Almería. Doctora en Ciencias de la Educación por la Universidad de Granada. Licenciada en Pedagogía y Graduada en Educación Infantil. Máster en Intervención psicopedagógica. Miembro del grupo de investigación FORCE (Formación del professorado centrada en la escuela- HUM-386), y de la Red de Investigación sobre Liderazgo y Mejora de la Educación (RILME). Sus líneas de investigación son la edu- 
cación inclusiva, la formación del profesorado, el liderazgo, las metodologías activas y las TIC. Email: mlinahr@ual.es

ORCID ID: https://orcid.org/0000-0002-4458-7339

\section{Ma del Mar García-Vita}

Licenciada en Historia y Doctora en Ciencias de la Educación. Docente del Departamento de Educación de la Universidad de Almería, en el área de Teoría e Historia de la Educación. Miembro Externo del Instituto de la Paz y los Conflictos de la Universidad de Granada. Sus temas de investigación se centran en la Pedagogía y la Educación Social y, en general, el trabaj desde el ámbito educativo con poblaciones vulnerables o en riesgo de exclusión. Algunos de sus últimos trabajos abordan temas de educación incusiva y educación para paz. Email: margvita@ual.es

ORCID ID: https://orcid.org/0000-0002-1710-5592 